\title{
Perfect practice makes perfect
}

\author{
Edward D. Matsumoto, MEd, MD, FRCSC \\ Associate Professor, Program Director Urology Residency, Director Laparoscopy and Endourology Fellowship; and Department of Urology, St. Joseph's Healthcare Institute of Urology, Hamilton, ON
}

See related article on page 331 .

Cite as: Can Urol Assoc J 2015;9(9-10):336. http://dx.doi.org/10.5489/cuaj.3360

Published online October 13, 2015.

commend the authors for implementing this surgical education study. ${ }^{1}$ With Competence by Design (CBD) on our doorstep, surgical educators across Canada will be making a significant transition to outcomes-driven and assessment-intense residency curricula within the next few years. For CBD to succeed, we must design and evaluate training and assessment tools that will enrich surgical residency curricula and ensure that residents become competent surgeons who can meet the expectations of patients and society. Due to time and financial constraints, surgical residency programs will have to rely more upon exvivo training, such as simulation, to prepare residents for the high-stakes operating room (OR) environment. It has been said time and time again that the fiscal constraints of the healthcare system are eroding valuable intra-operative teaching time. Preparing residents outside of the OR using low- and high-fidelity simulators allows them to acquire technical skills in a low-stakes environment that lends itself to deliberate practice and feedback as they move through the learning curve. This preparation should initiate a meaningful hands-on experience when residents transfer these skills to the OR. Basic skills, such as knot-tying, laparoscopic instrument handling, and even the use of endoscopic instruments, can be learned in an equipped surgical skills laboratory.

The authors have elegantly shown how a ureteroscopy curriculum can be developed by using a novel low-fidelity, relatively inexpensive simulated model. We should encourage discourse and advancement in surgical education research, including determining the most successful ways to prepare residents via simulation. We are only beginning to scratch the surface when it comes to assessing surgical residents outside and inside the OR.
I encourage the authors to look beyond the bench lab and consider the ultimate goal of our efforts as surgical educators - turning out competent surgeons. For this to be a methodical and valid endeavour, intra-operative assessment will be a critical step. Further efforts on development, refinement, and validation of assessment tools and methods of collecting, analyzing, and disseminating these data will be paramount. The OR remains a challenging environment to assess residents' surgical skills, as the priority is safe and effective patient care, as it should be. Innovative and nonintrusive methods of assessing surgical competency is highly desirable and therefore should be the focus of educators over the next few years as CBD is integrated into training. We have seen that training in a surgical skills laboratory leads to residents' improvement in the ex-vivo environment, but the question remains as to the extent to which this improvement transfers to the high-stakes OR environment.

For CBD to be successful in surgical residency, the assessment tools we use in the OR will need to be valid and reliable, and have the ability to be implemented in a safe manner without compromising patient care. As a surgeon educator, I look forward to the challenges of implementing CBD and ultimately improving the way we train future surgeons.

Competing interests: Dr. Matsumoto declares no competing financial or personal interests.

\section{Reference}

1. Blankstein U, Lantz AG, Honey RJ, et al. Simulation-based flexible ureteroscopy training using a novel ureteroscopy part-task trainer. Can Urol Assoc J 2015;9:331-5. http://dx.doi.org/10.5489/cuaj.2811

Correspondence: Dr. Edward Matsumoto, Associate Professor Urology, St. Josephs Healthcare Institute of Urology, 50 Charlton Ave E, Room G343, Hamilton, ON L8N 4A6; matsumo@mmaster.ca 